\title{
Protein Fem-1 Homolog C
}

National Cancer Institute

\section{Source}

National Cancer Institute. Protein Fem-1 Homolog C. NCI Thesaurus. Code C162440.

Protein fem-1 homolog C (617 aa, $\sim 69 \mathrm{kDa}$ ) is encoded by the human FEM1C gene. This protein is involved in E3 ubiquitin-protein ligase complex activity regulation. 